\title{
Réflexions autour de la laïcité axiologique
}

\author{
Lauren Bakir
}

\section{OpenEdition}

Journals

Édition électronique

URL : http://journals.openedition.org/rdr/435

DOI $: 10.4000 /$ rdr.435

ISSN : 2534-7462

\section{Éditeur}

Presses universitaires de Strasbourg

\section{Édition imprimée}

Date de publication : 14 novembre 2019

Pagination : 137-154

ISBN : 979-10-344-0054-6

ISSN : 2493-8637

\section{Référence électronique}

Lauren Bakir, « Réflexions autour de la laïcité axiologique », Revue du droit des religions [En ligne], 8| 2019, mis en ligne le 25 novembre 2019, consulté le 19 novembre 2020. URL : http:// journals.openedition.org/rdr/435; DOI : https://doi.org/10.4000/rdr.435

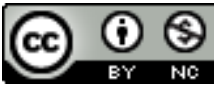

La revue du droit des religions est mise à disposition selon les termes de la Creative Commons Attribution - Pas d'Utilisation Commerciale 4.0 International - CC BY-NC 4.0. 


\section{RÉELEXIONSAUTOURDELA LÄ̈CITÉ AXIOLOGIQUE}

\section{Lauren BAKIR}

Docteure en droit public, Université de Strasbourg / CNRS, Droit, Religion, Entreprise et Société (DRES)

\section{RÉSUMÉ}

Si le principe de laïcité dispose d'un cadre juridique relativement clair en droit français, la laïcité conçue comme une valeur est abstraite et beaucoup plus difficile à cerner. L'étude d'une dizaine de textes relevant du droit souple, représentatifs du recours à la laïcité-valeur, permet d'en dresser les caractéristiques. Ces textes révèlent que la laiicité est à la fois envisagée comme une valeur politique et comme une valeur sociale. Toutefois, ni les textes de droit souple ni les autres corpus ne permettent de saisir le sens et les effets concrets de cette laïcité axiologique, plus affirmée qu'expliquée. Il apparaît que la laicité-valeur est une notion ambivalente qui peut renvoyer à des définitions aussi multiples que différentes. Deux hypothèses conceptuelles pourront être évoquées pour illustrer cette ambivalence.

\section{Abstract}

While the principle of laïcité has a relatively clear legal framework in French law, the value of laïcité is abstract and much more difficult to define. The study of about ten texts of soft law, representative of the use of laïcité as value, makes it possible to draw up its main features. These texts reveal that laicité is seen both as a political and as a social value. However, neither the texts of soft law nor the other text corpus allow us to grasp the meaning and concrete effects of this axiological laïcité, which is more assertive than explained. It appears that laicité as value is an ambivalent notion that can refer to definitions as multiple as they are different. Two conceptual hypotheses can be put forward to illustrate this ambivalence. 
ppréhendée sur le terrain politique, la notion de laïcité a un contenu
variable. Elle renvoie en effet à des conceptions politiques ou idéologiques de ce que sont, ou devraient être, les rapports de l'État et des religions. Sous cet angle, la laïcité cristallise des tensions et désaccords. Nombreux sont les auteurs à avoir qualifié, analysé et expliqué les différentes conceptions de la laïcité. Multiples et poreuses ${ }^{1}$, celles-ci ont évolué avec les contextes politiques et sociaux et sont difficiles à catégoriser ${ }^{2}$.

Sur le terrain du droit, la notion de laïcité est a priori plus claire. Elle peut être envisagée à travers la représentation pyramidale de l'ordre juridique proposée par Alexandre Viala. Selon cette représentation, le rapport entre les «valeurs», les «principes » et les «règles juridiques » est conçu «sous la forme d'une hiérarchie au-delà de laquelle les premières se trouvent reléguées dans une sphère qui relève de la philosophie morale à défaut d'intéresser la science du droit et au faîte de laquelle trônent les principes que les règles sont invitées à respecter ${ }^{3} »$. Dans ce schéma, les valeurs sont méta-juridiques et peuvent être définies comme «des émotions, des préférences qui ne sont ni vraies ni fausses et ne font l'objet d'aucune connaissance objective ${ }^{4} »$. Les principes sont le reflet des valeurs, ils s'insèrent dans le droit positif. Ils «gravent ces choix éthiques dans le marbre du droit positif au point de se présenter à la science du droit, par l'intermédiaire des énoncés qui en sont porteurs, comme un objet connaissable ${ }^{5} »$. Les règles juridiques sont quant à elles plus précises, elles «prescrivent, sous la forme d'énoncés dont la teneur déontique est explicite, des modèles de comportement auxquels les destinataires sont tenus de se conformer ${ }^{6} »$.

1. V. Notamment G. Bedouelle et J.-P. Costa, Les laïcités à la française, Paris, PUF, 1998; J. Bauberot, M. Milot, Ph. Portier (dir.), Laïcité, laïcités: reconfigurations et nouveaux défis. Afrique, Amériques, Europe, Japon, pays arabes, Paris, Éd. MSH, 2014; P. Flores D'ArCAIS, «Onze thèses sur la laïcité », Le Débat, 2015/3, p. 95; V. ZuBER, La laïcité en débat: au-delà des idées reçues, Paris, Le Cavalier bleu, 2017.

2. Jean Baubérot propose une cartographie de sept représentations de la laïcité, et distingue notamment les laïcités antireligieuse, gallicane, séparatiste, ouverte, identitaire, concordataire: J. BAuberot, Les sept laïcités françaises, Paris, Éd. MSH, 2015, p. 17 et s. Stéphanie Hennette-Vauchez et Vincent Valentin évoquent pour leur part les conceptions politiques de la laiicité, notamment libérale et républicaine: S. HennetTe-VAuchez, V. Valentin, L'affaire Baby Loup ou La nouvelle laïcité, Paris, LGDJ, 2014, p. 18.

3. A. Viala, «Valeurs et principes (distinction)», in J. Andriantsimbazovina et al. (dir.), Dictionnaire des droits de l'homme, Paris, PUF, 2008, p. 972.

4. Ibid.

5. Ibid.

6. Ibid. 
En droit français, le principe de laïcité occupe une place centrale. Principe d'organisation de la République ${ }^{7}$, il est consacré par le treizième alinéa du Préambule de la Constitution de 1946 et par l'article $1^{\text {er }}$ de la Constitution de 1958, puis précisé par le Conseil constitutionnel. En 2004, celui-ci interprète le principe de laïcité comme interdisant «à quiconque de se prévaloir de ses croyances religieuses pour s'affranchir des règles communes régissant les relations entre collectivités publiques et particuliers ${ }^{8} »$. En 2013, il précise que «le principe de laïcité figure au nombre des droits et libertés que la Constitution garantit» et «qu'il en résulte la neutralité de l'État; qu'il en résulte également que la République ne reconnaît aucun culte; que le principe de laïcité impose notamment le respect de toutes les croyances, l'égalité de tous les citoyens devant la loi sans distinction de religion et que la République garantisse le libre exercice des cultes; qu'il implique que celle-ci ne salarie aucun culte ${ }^{9} »$.

Le principe de laïcité se compose ainsi - en conformité avec les éléments proposés par le Conseil d'État dans son rapport Un siècle de laïcité publié en $2004^{10}$ et avec la doctrine ${ }^{11}$ - de trois éléments: la liberté religieuse, la neutralité de l'État et l'égalité entre les cultes.

Comme tout principe, et suivant en cela la pyramide proposée par Alexandre Viala, le principe de laïcité s'inspire des valeurs - libérales et humanistes ${ }^{12}$ - de la France et conduit à l'adoption de règles juridiques plus précises, comme l'obligation de neutralité des agents du service public ${ }^{13}$. C'est essentiellement le juge administratif qui a éclairé, progressivement, les contours du principe de laïcité et les modalités de sa mise en ouvre ${ }^{14}$.

7. La laïcité concerne les rapports entre les religions et l'État et signifie dans ce contexte «le refus de l'assujettissement du politique au religieux, ou réciproquement, sans être forcément synonyme d'étanchéité totale de l'un et de l'autre»: FRANCE. Conseil D'ÉtAT, Un siècle de laïcité, Paris, La Documentation française, 2004, p. 244-245.

8. Cons. const., déc. 19 nov. 2004, n² 2004-505 DC, Traité établissant une Constitution pour l'Europe, cons. 18.

9. Cons. const., déc. 21 févr. 2013, n 2012-297 QPC, Association pour la promotion et l'expansion de la laïcité, cons. 5.

10. France. Conseil d'État, Un siècle de laïcité, op. cit., p. 276.

11. V. notamment F. Messner, P.-H. Prelot, J.-M. Woehrling (dir.), Traité de droit français des religions, Paris, Litec, 2003, p. 48-53; D. Koussens, L'épreuve de la neutralité. La laïcité française entre droits et discours, Bruxelles, Bruylant, 2015, p. 28 et s.

12. A. Viala, «Valeurs et principes (distinction)», op. cit., p. 971.

13. CE, 3 mai 1950, Delle Jamet, $n^{\circ} 28238$. L'obligation de neutralité pesant sur les fonctionnaires est rappelée dans la loi $\mathrm{n}^{\circ}$ 2016-483 du 20 avril 2016 relative à la déontologie et aux droits et obligations des fonctionnaires.

14. Le juge administratif propose un traitement juridique pragmatique et adapté des multiples points de confluence entre le droit et les religions, V. notamment F. CurTit, « Le 
Ainsi, d'un point de vue strictement juridique, le principe de laïcité dispose d'un cadre relativement précis ${ }^{15}$.

En dépit de cela, et compte tenu notamment de l'évolution des problématiques religieuses dans une société sécularisée, le principe de laïcité et ses conséquences sur les comportements individuels sont parfois incompris de la société civile. À cet égard, le droit souple peut être une voie efficace pour éclairer et faciliter la mise en œuvre du régime juridique des rapports entre l'État et les religions ${ }^{16}$, et contribuer à l'acceptation de la norme par les destinataires visés.

Construction doctrinale, le droit souple a comme point de départ l'idée selon laquelle «le normatif ne se confond pas avec l'obligatoire ${ }^{17} »$. Dans cette perspective, un texte possédant une normativité, qu'elle soit plus ou moins dense ${ }^{18}$, est considéré comme une règle de droit ${ }^{19}$. Le droit souple, qui se manifeste dans toutes les branches du droit par le biais d'instruments très divers (avis, chartes, recommandations ${ }^{20} \ldots$ ) a vocation à inciter et à susciter l'adhésion: "La contrainte unilatérale est abandonnée au profit de la recherche d'une influence sur le comportement des individus, reposant sur leur adhésion ${ }^{21}$.»

droit français des religions au miroir des textes», in Droit et religion en Europe: études en l'honneur de Francis Messner, Strasbourg, Presses universitaires de Strasbourg, 2014, p. 63.

15. Les éléments constitutifs de ce principe peuvent évidemment être interprétés de façon variable: «la gouvernance politique ne va pas attribuer le même poids aux principes fondamentaux de la laïcité selon la nature du fait social auquel elle se trouve confrontée». David Koussens évoque à cet égard la «polymorphie matérielle» de la laïcité: D. Koussens, L'épreuve de la neutralité, op. cit., p. 36. V. également S. HennetTe-VAuchez, «Séparation, garantie, neutralité... Les multiples grammaires de la laïcité», N3C 2016/4, nº 53, p. 15. Un exemple: l'amendement adopté par le Sénat le 16 mai 2019 visant à interdire le port de signes religieux par les parents accompagnant les enfants lors de sorties scolaires.

16. F. Curtit et A. Fornerod, «Manifestations de la soft law en droit français des religions», Sciences religieuses, ${ }^{\circ} 2,2016$, p. 111.

17. C. Thibierge, « Le droit souple. Réflexion sur les textures du droit», RTD civ. 2003, p. 599.

18. À ce sujet, V. l'«échelle de densité normative» développée par Catherine Thibierge, ibid.

19. Cette démarche permet en effet de «rechercher ailleurs que dans la sanction le critère de la règle de droit et donc d'admettre que le droit souple puisse en faire partie», ibid.

20. Le droit souple se caractérise par une forte hétérogénéité: "de quelque côté que l'on se tourne, la soft law ne semble pas pouvoir être identifiée par un élément propre à en fournir le critère. La conclusion de cette quête de spécificité du droit souple tient toute entière dans un seul terme: pluralité. Pluralité des domaines et des sources, pluralité des instruments et des destinataires», ibid.

21. A. ForneroD, «Droit des religions et soft law», in Droit et religion en Europe: études en l'honneur de Francis Messner, op.cit., p. 102; V. également France. CONSEIL D'ÉTAT, Le droit souple, Étude annuelle 2013, Dossier de presse, p. 5. 
Dès lors, les autorités peuvent recourir au droit souple pour préciser et rendre accessibles certains principes juridiques. Par exemple, la Charte de la laïcité dans les services publics ${ }^{22}$ rappelle la signification du principe de laïcité et sa mise en ouvre dans les administrations. Les travaux de l'Observatoire de la laïcité, par le biais de rappels et de guides pratiques ${ }^{23}$, s'inscrivent également dans une démarche informative et pédagogique. Le principe de laïcité est précisé, mis en pratique, et rendu accessible aux citoyens. Publiés par des autorités diverses et possédant une normativité plus ou moins dense, ces textes ont pour point commun de s'inscrire dans une «pédagogie de la laïcité ${ }^{24} »$.

Toutefois, tous les textes de droit souple relatifs aux rapports entre l'État et les religions ne se limitent pas à éclairer le contenu et les effets du principe de laïcité. De nombreux textes de droit souple publiés ces dernières années dépassent ce cadre juridique en invoquant une laïcité axiologique, c'est-àdire une laïcité rattachée à la catégorie des «valeurs» mise en lumière par Alexandre Viala. Ce constat suscite deux remarques complémentaires. D'une part, présenter la notion de laïcité à la fois comme un principe et comme une valeur ne semble pas contribuer à une bonne compréhension du droit par les destinataires de ces textes. En effet, si le principe de laïcité dispose d'un cadre juridique précis, la laïcité-valeur est méta-juridique, donc abstraite par nature. D'autre part, cette qualification bouscule les cadres de référence traditionnels et perturbe la représentation pyramidale de l'ordre juridique, au sein de laquelle la laïcité est un principe ${ }^{25}$.

La laïcité-valeur investit par ailleurs d'autres corpus que le droit souple. D'un côté, elle est fréquemment mobilisée dans les déclarations et discours publics. Dans ce cadre, elle intègre le «récit lié à la laïcité ${ }^{26} »-$ qualifié de

22. Circ. n 5209/SG, 13 avr. 2007, Charte de la laicité dans les services publics.

23. Par ex. : ObSERVATOIRE DE LA LAḮcitÉ: Rappel du cadre légal général à propos de la laïcité et du fait religieux, juill. 2018; Rappel du cadre légal permettant de sanctionner les agissements contraires aux exigences minimales de la vie en société, févr. 2017.

24. M. Philip-Gay, Droit de la laïcité: une mise en ouvre de la pédagogie juridique de la laïcité, Paris, Ellipses, 2016.

25. La laiicité pourrait être qualifiée de «valeur» uniquement en adoptant une interprétation large de la notion de «valeurs de la République»: "Stricto sensu, ces valeurs sont incarnées par la devise Liberté égalité fraternité rappelée à l'article 2 de la Constitution; lato sensu, elles sont assimilées à l'ensemble des droits, libertés et principes de valeur fondamentale», dont fait partie la laïcité: M. Philip-Gay, «Les valeurs de la République», in Ph. Blacher (dir.), La Constitution de la Ve République: 60 ans d'application (1958-2018), Paris, LGDJ, 2018, p. 121. Si cette approche est riche sur le plan théorique, il pourrait néanmoins être risqué d'attribuer une normativité à cette notion encore insaisissable.

26. A. Ferrari, «De la politique à la technique: laïcité narrative et laïcité du droit. Pour une comparaison France/Italie», in B. BASDEVANT-GAUdEMET et F. JANKOWIAK (dir.), Le droit ecclésiastique de la fin du XVIII ${ }^{e}$ au milieu du XX $x^{e}$ siècle en Europe, Leuven, Peeters, 2009, p. 333-345. 
«laïcité narrative» dans la doctrine ${ }^{27}$ - et possède une normativité limitée. Elle pourra influencer l'adoption ou l'interprétation de certaines règles, mais toujours en conformité avec - ou dans les limites fixées par - le droit positif ${ }^{28}$. D'un autre côté, la laïcité-valeur apparaît également dans certains textes de droit dur, un droit "précis dans son contenu, obligatoire dans son expression et contraignant dans ses sanctions ${ }^{29}$ ». Certes, le recours explicite à la laïcitévaleur dans le droit dur demeure assez rare ${ }^{30}$. Néanmoins, la normativité de la laicité axiologique est alors aussi forte que problématique. Rien ne permet, en effet, de déterminer son sens et ses conséquences.

Dès lors, et bien que cette qualification de la laïcité comme valeur ne soit pas nouvelle ${ }^{31}$, sa récurrence au cours des dernières décennies interroge. Elle est en effet érigée au sommet de l'ordre juridique, devant donc fonder les principes et les modèles de comportement qui en découlent. Or, quel que soit le corpus sélectionné, le contenu de la laïcité-valeur et ses implications ne sont jamais précisés, laissant exister un doute profond sur ses déclinaisons concrètes.

Pour tenter de dresser les caractéristiques de la laïcité-valeur, les textes de droit souple constituent un corpus intéressant car abondants et hétérogènes par leur nature ${ }^{32}$, leurs origines ${ }^{33}$, et leurs destinataires ${ }^{34}$ (1). En revanche, pour cerner les implications découlant de cette qualification, l'étude des textes normatifs semble insuffisante. La laïcité-valeur y est en effet affirmée

27. Ibid.; V. également D. Koussens, L'épreuve de la neutralité, op.cit., p. 16.

28. Ph. Portier, L'État et les religions en France. Une sociologie historique de la laïcité, Rennes, Presses universitaires de Rennes, 2016, p. 69-71.

29. C. Thibierge, «Le droit souple. Réflexion sur les textures du droit», art. cit., p. 599.

30. Par ex.: art. 7 de la loi $n^{\circ}$ 2014-173 du 21 février 2014 de programmation pour la ville et la cohésion urbaine; $V$. aussi plusieurs décisions de la CAA Nantes, qui a une compétence spécifique dans le contentieux relatif à l'acquisition de la nationalité: CAA Nantes, 28 déc. 2015, $\mathrm{n}^{\circ}$ 15NT03371, cons. 4 ("pratique radicale de la religion incompatible avec les valeurs de tolérance et de laïcité de la société française»); 12 juin 2015, $n^{\circ}$ 14NT02533, cons. 3.

31. En 1995, Philippe Ardant notait que «la France est l'un des très rares pays où la laïcité soit posée comme un des principes fondateurs de l'État, comme une valeur républicaine complétant en quelque sorte la trilogie de 1789: liberté, égalité, fraternité, laïcité»: $\mathrm{Ph}$. Ardant, «La laïcité. Introduction», Pouvoirs, n 75, 1995, p. 5.

32. Chartes, rapports, livrets... Par ex.: Charte de la laïcité à l'école, 2013; Centre national DE LA FONCTION PUblique terRitoriale, La laïcité. Territoriaux, nous agissons, 2016.

33. Collectivités territoriales, personnes publiques... par ex.

34. Travail social, élèves des écoles publiques, fonctionnaires... V. aussi M. THIERRY, Valeurs républicaines, laïcité et prévention des dérives radicales dans le champ du travail social: rapport à la ministre des Affaires sociales, de la Santé et des Droits des femmes et à la secrétaire d'État chargée des Personnes handicapées et de la Lutte contre l'exclusion, 31 mars 2016. 
plus qu'expliquée. Aucun corpus ne permet de déterminer, pour le moment, les conséquences d'une telle place au sommet de l'ordre juridique. Le champ des possibilités est vaste: la laïcité-valeur pourrait aussi bien impliquer un maintien du principe de laïcité tel que consacré et mis en œuvre en droit français, que s'y opposer ${ }^{35}$. À l'aide des travaux de la doctrine, deux hypothèses conceptuelles pourront être proposées. Celles-ci attestent, du reste, de la forte ambivalence de cette notion (2).

\section{LA LAÏCITÉ-VALEUR AU PRISME DU DROIT SOUPLE}

L'étude d'une dizaine de textes de droit souple, non exhaustifs mais pas moins représentatifs du recours à la laïcité axiologique, en révèle deux dimensions indissociables: une dimension politique et une dimension sociale. La dimension politique se manifeste par l'adjonction de la laïcité aux valeurs de la République (1.1). Ainsi, soit les notions de «laïcité» et de «valeurs de la République» sont liées par la conjonction de coordination «et», soit la laïcité est présentée comme étant elle-même une des valeurs de la République. De façon concomitante, la laicité est présentée comme une valeur qui a vocation à être partagée par tous les individus vivant dans la société française. La laicité-valeur acquiert donc également une dimension sociale (1.2).

\subsection{L'ADJONCTION DE LA LAÏCITÉ AU TRIPTYQUE LIBERTÉ, ÉGALITÉ, FRATERNITÉ: LA LAÏCITÉ COMME VALEUR POLITIQUE}

Les différents textes de droit souple relatifs à l'appréhension du religieux publiés ces dernières années associent les notions de «laïcité » et de «valeurs de la République». Sans prétendre à l'exhaustivité, il est possible de mentionner la Charte de la laïcité à l'École ${ }^{36}$ - qui comporte, comme préambule, «La Nation confie à l'École la mission de faire partager aux élèves les valeurs de la République»-, la Charte régionale des valeurs de la République et de la laïcité d'Île-de-France ${ }^{37}$, la Charte de partage des valeurs républicaines adoptée

35. Selon Mathilde Philip-Gay, «on aurait tort de les opposer [le principe et la valeur de laiicité], ne serait-ce que parce que la valeur de laïcité nourrit le principe juridique qui en est le support. », M. Philip-Gay, «Les valeurs de la République», op. cit., p. 121.

36. France. Ministère de l'Éducation nationale et de la Jeunesse, Charte de la laïcité à l'école, 2013.

37. Île-de-France. Conseil RÉGIOnal, Charte régionale Île-de-France des valeurs de la République et de la laïcité, 2017. 
par la ville d'Elne, qui engage les responsables des associations signataires à «faire respecter, à promouvoir et à faire partager les valeurs découlant des principes de la devise républicaine et de la laïcité», la Charte du respect des valeurs de la République de la Région Provence-Alpes-Côte d'Azur qui vise moins directement la laïcité, et la Charte de la laïcité adoptée par la Caisse d'allocations familiales de Meurthe-et-Moselle qui vise moins spécifiquement les valeurs républicaines.

Il est également possible de se référer au rapport Valeurs républicaines, laïcité et prévention des dérives radicales dans le champ du travail social ${ }^{38}$ - qui répond à une lettre de mission de la ministre des Affaires sociales, de la Santé et des Droits des femmes, Marisol Touraine, et qui doit apporter des réponses à différentes questions formulées par la ministre, à travers lesquelles la laïcité apparaît comme une valeur de la République ${ }^{39}$ - ou encore au rapport Laïcité, valeurs de la République et exigences minimales de la vie en société ${ }^{40}$ remis au ministre de l'Intérieur.

Le rapport La laïcité. Territoriaux, nous agissons du Centre national de la fonction publique territoriale (CNFPT) appréhende la laïcité comme un « débat républicain ${ }^{41} »$. Si la majeure partie des encadrés de ce rapport - rédigés par les collectivités territoriales ${ }^{42}$ - appréhendent la laïcité comme principe juridique ${ }^{43}$, l'éditorial publié en première page de ce rapport ${ }^{44}$ indique que les élus et agents publics «sont les premiers garants de la mise en œuvre de nos valeurs républicaines, à commencer par celle de la laïcité ${ }^{45} »$. Il en est de même dans l'introduction du livret numérique Les fondamentaux sur la

38. M. ThIERry, Valeurs républicaines, laïcité et prévention des dérives radicales dans le champ du travail social, op. cit.

39. Ibid., p. 5.

40. G. Clavreul, Laïcité, valeurs de la République et exigences minimales de la vie en société. Des principes à l'action: rapport au ministre de l'Intérieur, févr. 2018. Pour une analyse de ce rapport, V. notamment S. DeGiRMENCI, «Le rapport Clavreul ou les errements d'un certain discours sur la laïcité», Rev. DH 2018: http://journals.openedition.org/revdh/3805 [consulté le 13 juin 2019].

41. Et un «principe républicain avec un enjeu sociétal et politique fort»: CENTRE NATIONAL DE LA FONCTION PUblique TeRritoriale, La laïcité. Territoriaux, nous agissons, 2016, p. 4.

42. Ce livret contient en effet les actions locales clés «qui témoignent de l'esprit d'initiative des collectivités territoriales et de leur volonté de prendre à bras-le-corps la question de la laïcité» : ibid., p. 4.

43. La mairie de Montpellier rappelle que la laïcité est un principe et implique une neutralité des agents «car c'est la République qui est laïque, et non la société»: ibid., p. 5.

44. Rédigé par M. François Deluga, président du Centre national de la fonction publique territoriale (CNFPT) et maire du Teich.

45. «Les collectivités s'emparent de la question de la laïcité»: ibid., p. 3. 
laïcité et les collectivités territoriales disponible sur le site du $\mathrm{CNFPT}^{46}$, dans lequel il est indiqué que la laïcité «qui a guidé la loi de séparation [...], et a été inscrite dans les Constitutions de 1946 et de 1958, incarne désormais l'une des principales valeurs républicaines ${ }^{47}{ }^{»}$.

Le livret Points de repère pour l'exercice des responsabilités ${ }^{48}$ adjoint également la laïcité-valeur à la laïcité-principe: la première partie du livret rappelle «quelles sont les valeurs et les responsabilités des agents de la fonction publique territoriale. La laïcité y est décrite comme une valeur de la République, mais également comme une obligation liée au statut de fonctionnaire», tandis que la deuxième partie (Guide pratique) rappelle «ce qu'est ce principe fondateur de notre République».

Enfin, sur le site Internet du réseau Canopé ${ }^{49}$, la laïcité est classée parmi les valeurs de la République. Certes, il s'agit d'un site de ressources pédagogiques qui s'adresse aux enseignants, mais la laïcité axiologique y est mentionnée dans la perspective de sa diffusion à tous les enseignants et élèves, invités à y adhérer. Ces ressources possèdent donc également, bien que plus indirectement, une normativité. Comme pour les autres valeurs évoquées, un onglet est consacré à la laïcité axiologique pour l'expliciter ${ }^{50}$. Dans la sous-partie «Poursuivre la réflexion philosophique», rédigée par le professeur de philosophie Michel Delattre, la laiicité apparaît explicitement comme une valeur de la République: «si on entend par neutralité le fait de rester indifférent, de ne pas intervenir, on voit que la laïcité est une valeur plus forte, un véritable engagement. C'est une valeur républicaine qui affirme

46. http://www.cnfpt.fr/sites/default/files/laicite-web3_0.pdf [consulté le 13 juin 2019].

47. Centre national de la fonction publique territoriale, La laïcité. Territoriaux, nous agissons, op.cit., p. 3 [souligné par nous]. V. également l'intitulé du rapport de l'Inspection générale de l'Éducation nationale, Séminaire des doyens: Valeurs de la République et laïcité, 15-16 avril 2015 et l'avant-propos de Jean-Yves Daniel, p. 1, qui évoque la «dimension intimement laïque si spécifiquement française» des valeurs républicaines.

48. Ce livret fait l'objet d'une présentation dans Centre nATIONAL DE LA FonCtion publique TERRITORIALE, La laïcité. Territoriaux, nous agissons, op.cit., p. 13.

49. C'est un réseau de création et d'accompagnement pédagogiques placé sous la tutelle du ministère de l'Éducation nationale: https://www.reseau-canope.fr/les-valeurs-de-larepublique/les-valeurs-et-notions.html [consulté le 13 juin 2019].

50. Dans l'onglet consacré à la laïcité, figurent notamment un extrait de: B. STASI, «La laïcité», in Centre national de documentation pédagogiQue, Guide républicain. L'idée républicaine aujourd'hui, Paris, Delagrave, 2004; J. GRONDEUX, «La laïcité scolaire», qui se réfère notamment aux lois Ferry et à la loi du 15 mars 2004 et "La République laïque », qui se réfère notamment à la loi du 9 décembre 1905 et aux articles des constitutions de 1946 et 1958; M. Delattre, «Poursuivre la réflexion philosophique»; O. Loubes, «Poursuivre la réflexion historique: la laïcité». 
des droits qui sont des conditions d'existence en commun $[\ldots]^{51}$.» Il faut également souligner que, dans l'onglet consacré à la valeur «Fraternité », les valeurs de fraternité et de laïcité sont assimilées.

La fraternité est ainsi présentée comme «l'enfant naturelle de la République », «la liberté et l'égalité, les grandes aînées, se mettent en droit, font leur lit dans la loi [...]. La fraternité, valeur morale mais non juridique » vient de «ce qui relie les hommes, elle est la religion en somme (religere, 'ce qui relie'). Religion civique, la fraternité est la morale de l'histoire républicaine, celle qui, allant vers les autres, permet de dire que notre société a réussi sa composition française. À l'âge des nations - devenues corps social et politique de l'État -, c'est par elle que l'on mesure la qualité de cohésion de la communauté nationale. À l'aune de l'histoire française de la constitution de l'État et de la nation en République, le cadre de cette cohésion fraternelle, émancipatrice, a pris le nom de laïcité, depuis l'article 10 de la Déclaration des droits de l'homme et du citoyen de 1789 jusqu'aux réglages de 1905 et $2004^{52}$ ».

Ces différents exemples mettent en lumière que la laïcité n'est plus uniquement envisagée comme un principe d'organisation de la République, ou comme un vecteur des valeurs de la République, elle devient elle-même une valeur de la République.

\subsection{L'ADJONCTION DE LA LAÏCITÉ AUX VALEURS DE LA SOCIÉTÉ : LA LAÏCITÉ COMME VALEUR SOCIALE}

La laïcité-valeur est présentée, dans les différents documents étudiés, comme une valeur qui a vocation à être partagée par tous les individus, ce qui s'illustre à la fois par le nombre important de documents publiés en ce sens et la diversité des espaces communs concernés.

Dans le milieu scolaire, l'article 9 commenté de la Charte de la laïcité à l'école indique que "la laïcité n'est pas seulement un principe qui impose des règles, mais une valeur à cultiver dans les conduites au quotidien ${ }^{53}$ ». La circulaire à laquelle la Charte est annexée mentionne également le «principe de laïcité à l'École» et la laïcité comme «valeur positive d'émancipation ${ }^{54}$.

51. Ibid.

52. Ibid. [souligné par nous].

53. Charte de la laïcité à l'école, 2013, et V. l'art. 10 qui se réfère à la «valeur de laïcité».

54. Circ. $n^{\circ}$ 2013-144, 6 sept. 2013, relative à la Charte de la laïcité à l'école et aux valeurs et symboles de la République. 
Dans le domaine des aides sociales, il est possible de se référer à la Charte de la laïcité de la Caisse d'allocations familiales de Meurthe-et-Moselle qui indique que "porteurs d'éducation populaire, les centres sociaux se doivent de transmettre le sens et la valeur de la laïcité [...] à leurs adhérents et à l'ensemble de leurs équipes». La valeur laïcité est également portée dans le domaine du travail social. Ainsi le rapport Valeurs républicaines, laïcité et prévention des dérives radicales dans le champ du travail social évoque-t-il les «nouveaux défis pour un exercice laíc du travail social ${ }^{55} »$. Dans ces deux exemples, la laïcité axiologique est donc portée et diffusée par les acteurs sociaux, élargissant ainsi son spectre de diffusion.

Les usagers des services publics connaissent également cette valeur de laicité. Dans les quelques lignes rédigées par François Deluga en introduction du livret numérique Les fondamentaux sur la laïcité et les collectivités territoriales ${ }^{56}$, il précise que le fonctionnaire est soumis à un devoir de neutralité (justifié par l'égalité) et que «les usagers des services publics, quant à eux, ont des obligations au regard de la valeur de laïcité $[\ldots]^{57} »$.

Eu égard à la diversité des destinataires invités à adhérer à la laïcité-valeur, elle acquiert une place importante dans la vie en société. En tant que valeur sociale, ses effets sont diffus. D'une part, l'idée selon laquelle la laïcité est une valeur est introduite dans l'imaginaire commun. D'autre part, et de façon concomitante, la laïcité axiologique a vocation à déployer ses effets dans les relations interindividuelles et, plus généralement, dans tout l'espace social.

Toutefois, la laicité-valeur est affirmée, proclamée, plus qu'expliquée. Il est donc difficile d'en déterminer les effets concrets ou d'en donner des exemples. Dès lors, en l'absence de précisions quant au contenu et aux implications de la laïcité axiologique, cette référence semble poser plus de questions qu'elle n'en résout. En 1995, dans le contexte de l'adoption de l'article 8 de la loi constitutionnelle du 4 août $1995^{58}$, Philippe Ardant relevait déjà que la laïcité fut l'objet de vives oppositions et s'interrogeait: "comment ne pas relever en même temps que, curieusement, l'objet des combats est resté

55. M. THIERry, Valeurs républicaines, laïcité et prévention des dérives radicales dans le champ du travail social, op. cit.

56. Disponible sur le site du CNFPT: http://www.cnfpt.fr/sites/default/files/laicite-web3_0. pdf [consulté le 13 juin 2019].

57. Centre national de la fonction publique territoriale, Les fondamentaux sur la laïcité et les collectivités territoriales, op. cit., "Édito», p. 4.

58. Cet article 8 abrogeait l'article $1^{\text {er }}$ de la Constitution, remplacé par le premier alinéa de l'article 2 selon lequel «La France est une République indivisible, laique, démocratique et sociale» : L. const. n 95-880, 4 août 1995. 
imprécis tant était incertaine la conception même de la laïcité? ${ }^{59} »$. Compte tenu de la récurrence du recours à la laïcité-valeur ces dernières décennies, cette question a encore toute sa pertinence aujourd'hui.

Certes, dans la définition lato sensu des «valeurs de la République» proposée par Mathilde Philip-Gay, la laïcité peut être appréhendée à la fois comme un principe et comme une valeur de la République ${ }^{60}$. Néanmoins, et ainsi qu'elle le relève, se pose nécessairement la question «de leur conciliation sous cette double forme ${ }^{61} » . \mathrm{Si}$, sur le terrain de la théorie, cette qualification de la laïcité n'est pas problématique, elle le devient lorsque les conséquences concrètes du recours à la laïcité-valeur sont difficiles à mesurer. Sa récurrence, et l'invitation faite aux individus d'y adhérer, interrogent nécessairement. En l'absence de précisions quant à son contenu - dans les textes de droit souple comme dans les autres corpus juridiques - quels sont ses implications et ses effets?

\section{L'AMBIVALENCE DE LA LAÏCITÉ-VALEUR}

L'érection de la laïcité en valeur bouleverse les cadres de référence traditionnels en matière de prise en compte du religieux. Envisagée comme une valeur, la laïcité investit le sommet de l'ordre juridique et interroge quant à sa portée. Le principe de laïcité et les règles juridiques qui en découlent sont-ils conformes, dans leur interprétation actuelle, à cette laïcité-valeur? Ou cette notion a-t-elle vocation à modifier, sur le long terme, l'état du droit positif? Les textes normatifs actuels se référant à la laicité axiologique la proclament sans jamais expliciter son sens ou ses implications. Dès lors, la palette des possibilités conceptuelles est très large. Éclairées par les travaux de la doctrine, deux hypothèses peuvent être proposées. Dans la première, la laïcité-valeur s'inscrit dans un lien très étroit avec la vie en société. Elle représente un socle axiologique commun, selon lequel les individus ne peuvent s'exclure de la vie en société en raison de leur appartenance religieuse (2.1). Dans ce cadre, non seulement elle est conforme au principe de laïcité, mais elle le complète. Dans la seconde hypothèse, la laïcité-valeur a également vocation à assurer la vie en société mais, plus fortement encore, elle incarne un dogme ${ }^{62}$, une «religion

59. Ph. ARdant, «La laïcité. Introduction», art. cit., p. 5.

60. M. Philip-Gay, «Les valeurs de la République», op.cit., p. 121.

61. Ibid.

62. Jean Baubérot indique, à l'égard des affirmations de la laïcité gallicane, qu'elles « relèvent d'un "dogme" d'une religion civile, non d'un savoir validé par les sciences humaines » et rapproche cette conception de la conception actuelle des «valeurs républicaines» qui 
civile ${ }^{63}$ à laquelle les individus doivent adhérer. Dans cette perspective, et compte tenu des multiples polémiques relatives à l'extériorisation des convictions religieuses dans les espaces communs ${ }^{64}$, la laïcité-valeur pourrait avoir pour effet de circonscrire la visibilité religieuse à la sphère privée ${ }^{65}(2.2)$. Dès lors, elle entrerait en opposition avec le principe de laïcité tel que consacré en droit français.

\subsection{LA LAÏCITÉ-VALEUR : UNE LIMITE AUX COMPORTEMENTS D'EXCLUSION SOCIALE MOTIVÉS PAR DES CONSIDÉRATIONS RELIGIEUSES}

L'évolution des problématiques religieuses en France remet à l'ordre du jour les débats autour de la pratique de la religion. Si certaines expressions de la religion relèvent des qualifications prévues par le droit ${ }^{66}$, toutes ne peuvent être réglementées, sauf à uniformiser et homogénéiser les comportements individuels. Il n'en demeure pas moins que certains phénomènes - comme le «communautarisme» ou le repli identitaire - et certains comportements - comme le rejet de la mixité ou le refus de serrer la main à une personne de sexe opposé - peuvent perturber la vie en société. En créant, de façon plus ou moins prononcée, une rupture avec autrui, ces phénomènes et comportements troublent la vie en société.

Dès lors, la laïcité axiologique pourrait être conçue comme le versant sociétal du principe de laïcité. Là où le principe de laïcité emporte la neutralité religieuse des agents du service public et la liberté religieuse des personnes privées, la laïcité-valeur s'adresserait essentiellement aux personnes privées dont la pratique religieuse laisse penser qu'elle les exclut de la vie en société.

sont «dogmatiquement énoncées»: J. BAUBEROT, Les sept laïcités françaises, op.cit., p. 48 et p. 50 .

63. J. Bauberot, «Existe-t-il une religion civile républicaine?», French Politics, Culture E Society 2007, p. 3 et s.

64. V. infra.

65. V. Zuber, La laïcité en débat: au-delà des idées reçues, op.cit., p. 79 («Les religions doivent être reléguées à l'espace privé»); Ph. ARDANT, «La laïcité. Introduction», art. cit., p. 5 («morale sociale»); V. également N. KADA, «Service public et religion: du renouveau du principe de neutralité», AJFP 2004, p. 249 qui évoque une «laïcité prescriptive».

66. Par ex. : à l'école, pour les agents des services publics, ou encore dans l'entreprise, V. les lois no 2004-228 du 15 mars 2004 encadrant, en application du principe de laïcité, le port de signes ou de tenues manifestant une appartenance religieuse dans les écoles, collèges et lycées publics; $\mathrm{n}^{\circ} 2016-483$ du 20 avril 2016 relative à la déontologie et aux droits et obligations des fonctionnaires; $n^{\circ} 2016-1088$ du 8 août 2016 relative au travail, à la modernisation du dialogue social et à la sécurisation des parcours professionnels. Ces lois encadrent, à différents degrés et pour des raisons différentes, l'extériorisation des convictions religieuses. 
Dans ce schéma, la laïcité-valeur pourrait être envisagée comme le pendant axiologique de la décision du Conseil constitutionnel n²004-505 DC du 19 novembre 2004, relative au Traité établissant une Constitution pour l'Europe, selon laquelle «les dispositions de l'article $1^{\text {er }}$ de la Constitution aux termes desquelles "la France est une République laïque" [...] interdisent à quiconque de se prévaloir de ses croyances religieuses pour s'affranchir des règles communes régissant les relations entre collectivités publiques et particuliers » (cons. 18). Dans la même perspective, la laïcité-valeur pourrait interdire à quiconque de se prévaloir de ses croyances religieuses pour s'affranchir des valeurs communes régissant les relations entre les particuliers.

Par exemple, en France, si l'égalité des sexes est un principe juridique, elle est également une valeur sociale largement partagée, dont les effets se manifestent au quotidien. Deux cas de figure peuvent être distingués. D'une part, au regard de la décision précitée du Conseil constitutionnel de 2004, un individu ne pourrait se prévaloir de ses croyances religieuses pour s'affranchir du principe d'égalité des sexes. D'autre part, dans le cadre d'une approche axiologique de la laïcité, un individu ne pourrait se prévaloir de ses croyances religieuses pour justifier une atteinte à la valeur d'égalité des sexes. Si un individu refuse de serrer la main à un individu du sexe opposé, il ne contrevient pas au principe juridique d'égalité des sexes ${ }^{67}$, mais il crée bien un trouble dans sa relation à autrui et, de ce fait, dans la vie en société. La laïcité-valeur aurait alors vocation à reléguer à la sphère privée les comportements ou actes de nature religieuse qui entrent en opposition frontale avec les valeurs sociales, et de préserver les conditions de la vie en société ${ }^{68}$.

La « laïcité existentielle ${ }^{69}$ » développée par Abdennour Bidar, et qui s'inspire notamment des écrits de Jean Jaurès ${ }^{70}$, semble s'inscrire en ce sens. D'une part, l'accent est mis sur les notions de laïcité et de démocratie ${ }^{71}$ : "Laïcité et démocratie sont identiques", écrivait [Jean Jaurès], ou "indivisibles", parce que la séparation laiqque du religieux et du politique est en réalité consubs-

67. Refuser de serrer la main à une personne du sexe opposé n'est pas pénalisé par le droit positif.

68. En ce sens: «La laïcité, comme philosophie porteuse d'un idéal de dépassement des identités particulières et de neutralisation des "guerres de religion", devrait pouvoir irriguer l'ensemble des relations humaines, dans et en dehors de l'État»: V. VALEnTin, «Laïcité et neutralité», AJDA 2017, p. 1388.

69. A. Bidar et al., "Que veut dire laïcité? Enquête», Le Débat 2015/3, p. 104.

70. Abdennour Bidar cite notamment les explications données par Jean Jaurès dans le journal L'Humanité le 2 août 1904: ibid.

71. V. également M. Wieviorka, «Laïcité et démocratie», Pouvoirs, n 75, nov. 1995, p. 61. 
tantielle à une "démocratie" qui "fonde en dehors de tout système religieux toutes ses institutions, tout son droit politique et social, famille, patrie, propriété, souveraineté" $72 »$. D'autre part, et de façon complémentaire, un lien est établi entre les notions de laïcité et de démocratie et les notions d'égale dignité des personnes et de respect mutuel: «La laïcité dite française ne fait que concrétiser de la façon la plus nette, la plus achevée, le principe d'une démocratie qui "ne s'appuie que sur l'égale dignité des personnes humaines appelées aux mêmes droits et invitées à un respect réciproque" et qui, par conséquent - c'est toujours Jaurès qui parle -, "se dirige sans aucune intervention dogmatique et surnaturelle, par les seules lumières de la conscience et de la science" 73 ». Ce raisonnement, qui rappelle «l'ambition humaniste ${ }^{74}$ » de la laïcité, pourrait orienter la définition qu'il convient de donner à la laïcité-valeur et l'enrichir. Envisagée sous ce prisme, la laïcité-valeur pourrait en effet incarner à la fois le lien vertical existant entre l'individu et la communauté politique et le lien horizontal existant entre l'individu et le corps social. Sous cet angle, la relation entre la laicité-valeur et la vie en société est forte. L'appartenance au corps social impliquerait l'impossibilité pour les individus de s'exclure de la vie en société pour des motifs religieux, ainsi que le respect des valeurs sociales communes ${ }^{75}$.

Ainsi, les individus seraient invités non pas tant à adhérer à la laïcité axiologique qu'à la respecter, voire à la partager, dans le but de préserver les conditions essentielles à la vie en société. Dans cette perspective, la laïcité axiologique viendrait compléter, pour répondre à l'évolution des pratiques religieuses, le principe de laïcité.

\subsection{LA LAÏCITÉ-VALEUR : UNE « RELIGION CIVILE » 76}

Sous un angle strictement juridique, la laïcité est un principe dont le cadre est défini et qui permet la « coexistence de divers régimes de croyance ${ }^{77} »$. En

72. A. BidAR et al., art. cit.

73. Ibid.

74. Ibid.

75. À propos de ce raisonnement, V. F. SAINT-BonnET, «La citoyenneté, fondement démocratique pour la loi anti-burqa», Jus Politicum, n 7, 2012: http://juspoliticum.com/ article/La-citoyennete-fondement-democratique-pour-la-loi-antiburqa-452.html [consulté 23 juin 2019].

76. J. BAuberot, «Existe-t-il une religion civile républicaine?», art. cit.; V. également Les sept laïcités françaises, op. cit., p. 37.

77. S. Hennette-Vauchez, «Séparation, garantie, neutralité... Les multiples grammaires de la laïcité», art. cit., p. 18. 
revanche, érigée en valeur, la laïcité devient une "émotion, [une] préférence » qui ne fait l'objet «d'aucune connaissance objective ${ }^{78}$. Elle «acquiert un contenu substantiel et véhicule une conception du bien ${ }^{79}$ ». Jean Baubérot explique en ce sens que «chaque fois que l'on invoque un principe "républicain" [...] en le transformant en une "valeur" à laquelle il convient d'adhérer, sans mettre en débat son interprétation et sa concrétisation, on adopte une perspective de religion civile: elle consiste à fonder une "société républicaine" sur une "transcendance qui se dérobe au jugement" 80 ». Dès lors, elle dépasse le seul objectif de vie en société, elle devient elle-même un «projet de société ${ }^{81}$ ». Les individus sont donc appelés à adhérer à «une définition de la vie bonne, une manière d'être ${ }^{82}$ » qui emporte deux conséquences.

Sur le plan axiologique, une friction peut se créer entre la laicité-valeur, à laquelle les individus doivent adhérer, et leurs convictions religieuses. Il peut en résulter une concurrence, voire une prévalence de la laïcité-valeur sur les valeurs religieuses. En ce sens, Jean-Jacques Rousseau évoquait, dans sa Lettre à Voltaire: "un Code moral ou une espèce de profession de foi civile, qui contînt positivement les maximes morales que chacun serait tenu d'admettre, et négativement les maximes fanatiques qu'on serait tenu de rejeter, non comme impies, mais comme séditieuses. Ainsi toute religion qui pourrait s'accorder avec le Code serait admise; toute religion qui ne s'y accorderait pas serait proscrite; et chacun serait libre de n'en avoir point que le Code lui-même ${ }^{83}$ ».

Sur le plan pratique, la laïcité-valeur peut impliquer un cantonnement de l'extériorisation de la religion à la sphère privée. Il ne s'agirait alors plus uniquement d'une laïcité de l'État, mais d'une laïcisation de la société dans

78. A. Viala, «Valeurs et principes (distinction)», op. cit., p. 972.

79. S. Hennette-Vauchez, «Séparation, garantie, neutralité... Les multiples grammaires de la laïcité», art. cit., p. 18. Les partisans de cette laïcité sont notamment Henri Peña-Ruiz, Guy Coq, Régis Debray, Catherine Kintzler. V. D. Koussens, L'épreuve de la neutralité. La laïcité française entre droits et discours, op.cit., p. 43-45. V. également F. de LA MorenA, Les frontières de la laïcité, Paris, LGDJ, 2016.

80. J. Bauberot, Les sept laïcités françaises, op. cit., p. 37. Dans le même sens, David Koussens indique que la laïcité est ainsi porteuse de valeurs républicaines, ce qui signifie qu'elle véhicule une certaine conception de ce qui est bien: D. Koussens, L'épreuve de la neutralité. La laïcité française entre droits et discours, op.cit., p. 44-46.

81. V. Valentin, «Remarques sur les mutations de la laïcité. Mythes et dérives de la "séparation" », RDLF 2016, chron. ${ }^{\circ}$ 14, p. 7: http://www.revuedlf.com/droit-administratif/ remarques-sur-les-mutations-de-la-laicite-mythes-et-derives-de-la-separation/ [consulté le 13 juin 2019].

82. Ibid.

83. Cité par J. Bauberot, Les sept laïcités françaises, op.cit., p. 36. 
son ensemble - ce qui constitue pour le chercheur «une absurdité $e^{84} »$. La religion ne pourrait alors se manifester ni dans les comportements individuels ni dans les relations intersubjectives. Bien sûr, actuellement, une telle acception entre en opposition évidente avec le cadre juridique des rapports entre l'État et les religions. Celui-ci garantit, aux niveaux national, européen et international, la liberté religieuse des personnes privées dans les limites prévues par le droit. Néanmoins, il est difficile de ne pas constater que le recours à la laïcité-valeur ces dernières années est concomitant avec un contexte politique et social tendu à l'égard de la visibilité religieuse. En février 2018, l'«affaire Mennel», une jeune candidate portant un turban lors de son passage dans l'émission The Voice ${ }^{85}$, en mai 2018, la controverse au sujet de Maryam Pougetoux, «la responsable voilée de l'Unef ${ }^{86}$ », en février 2019, l'affaire du «hijab de Décathlon ${ }^{87}$ », en mai 2019, l'amendement voté par le Sénat visant notamment à interdire le port du voile aux mères accompagnant les enfants lors de sorties scolaires ${ }^{88}$, ne sont que quelques exemples des crispations politiques et sociales existant autour de la visibilité religieuse dans les espaces communs. Dans ce contexte, il semble primordial de définir ou, tout du moins, de préciser les contours de la laicité-valeur avant de la mobiliser dans des textes de droit.

84. Valentine Zuber distingue «la laïcité comme instrument politique, de la sécularisation qui qualifie la plus ou moins grande distance des valeurs portées socialement vis-à-vis des religions et de leurs expressions publique ou privée. La laïcité ne peut donc pas qualifier une société (pour le chercheur parler de société laïque est une absurdité...), mais simplement un système politico-juridique (la République est laïque)»: V. ZubER, La laïcité en débat: au-delà des idées reçues, Paris, Le Cavalier bleu, 2017, p. 12.

85. "The Voice": 4 actes pour comprendre l'affaire Mennel», L'Obs, 12 févr. 2018: https:// teleobs.nouvelobs.com/actualites/20180212.OBS2067/the-voice-4-actes-pour-comprendrel-affaire-mennel.html [consulté le 13 juin 2019].

86. M. Aublanc, «Qui est Maryam Pougetoux, la responsable voilée de l'Unef? », 20 minutes, 23 mai 2018: https://www.20minutes.fr/societe/2276127-20180523-maryam-pougetouxresponsable-voilee-unef [consulté le 13 juin 2019].

87. C. Pina, "Hijab de Décathlon: "Voilà pourquoi nous ne devons pas reculer face aux islamistes" », Le Figaro, 27 mai 2019: http://www.lefigaro.fr/vox/societe/2019/02/27/3100320190227ARTFIG00093-hijab-de-decathlon-voila-pourquoi-nous-ne-devons-pas-reculerface-aux-islamistes.php [consulté le 13 juin 2019].

88. F. Vignal, «Sorties scolaires: le Sénat vote pour l'interdiction du port du voile pour les mères accompagnatrices», Public Sénat, 17 mai 2019: https://www.publicsenat.fr/article/ parlementaire/sorties-scolaires-le-senat-vote-pour-l-interdiction-du-port-du-voile-pour-les [consulté le 13 juin 2019]. 


\section{CONCLUSION}

Adoptés sous des gouvernements successifs, les textes de droit souple proclament la laïcité-valeur de façon récurrente. Quels que soient les autorités ayant publié ces textes, la nature de ces textes ou leurs destinataires, le traitement de la notion de laïcité est identique: elle apparaît à la fois comme un principe et comme une valeur. En tant que valeur politique, la laïcité a vocation à inspirer les principes et les modèles de comportement qui en découlent. Dans sa dimension sociale, la laïcité-valeur peut être appréhendée comme un complément à sa dimension politique ${ }^{89}$. En effet, les textes de droit souple invitent tous les individus à adhérer et à partager cette laïcité axiologique. Ces textes, comme les autres corpus se référant à la laïcité-valeur, ne précisent ni son contenu ni ses effets. Dès lors, et à l'aide des travaux de la doctrine, deux hypothèses conceptuelles peuvent être proposées. Dans la première, la laïcité axiologique incarne un socle commun favorisant la vie en société. Ainsi envisagée, elle apparaît comme «l'affirmation universaliste que l'on est d'abord un être humain et un citoyen avant d'être quoi que ce soit d'autre ${ }^{90} »$. Dans la seconde hypothèse, la laïcité axiologique dépasse le seul objectif de vie en société, elle devient une «religion civile» à laquelle il faut adhérer. Dans cette hypothèse, une concurrence peut se créer entre valeurs religieuses et laïcité-valeur. Or, cette concurrence comporte le risque de faire de la laïcité une "valeur rempart ${ }^{91}{ }$ difficilement conciliable avec le cadre juridique actuel du principe de laïcité.

89. Les valeurs sociales et politiques ne sont pas, en effet, systématiquement identiques. V. notamment C. LE BRIS, «La contribution du droit à la construction d'un "vivre ensemble" : entre valeurs partagées et diversité culturelle», Droit et société, $\mathrm{n}^{\circ} 1,2016$, p. 75: "Les valeurs dites "républicaines" [...] ne peuvent être considérées comme épuisant les valeurs "communes".»

90. Ph. Gaudin et al., «Que veut dire laïcité? Enquête», Le Débat 2015/3, n 185, p. 110.

91. M. Philip-Gay, «Les valeurs de la République», op. cit., p. 121 